\title{
The non-targeted metabolomic profiling of liver reveals the molecular perturbations underlying clonorchiasis
}

\author{
Xiaoli Zhang \\ Harbin Medical University \\ Ruifeng Wang \\ Harbin Medical University Fourth Hospital \\ Beibei Sun \\ Harbin Medical University \\ Rui Chen \\ Harbin Medical University Fourth Hospital \\ Xinyi Hu \\ Harbin Medical University \\ Yannan Gao \\ Harbin Medical University \\ Shanshan Duan \\ Harbin Medical University \\ Jing Fang \\ Harbin Medical University Fourth Hospital \\ Ping Wang \\ Harbin Medical University \\ Xinshu Zhao \\ Harbin Medical University \\ Su Han ( $\sim$ hshshs1982@126.com ) \\ Harbin Medical University
}

\section{Research article \\ Keywords: Clonorchis sinensis, infection, liver, non-targeted metabolomics, metabolic pathway}

Posted Date: June 22nd, 2020

DOI: https://doi.org/10.21203/rs.3.rs-35184/v1

License: (c) (i) This work is licensed under a Creative Commons Attribution 4.0 International License. Read Full License 


\section{Abstract}

Background: Clonorchiasis is a common food-borne parasitic disease worldwide. However, the molecular mechanisms of host-parasite interaction has not been illustrated. Given the parasite could influence host metabolism, the hepatic metabolic profiling will be useful for exploring the pathogenic mechanism of clonorchiasis.

Results: The global hepatic metabolic profile of male Wistar rats at 4 and 8 weeks post infection with Clonorchis sinensis were investigated using liquid chromatography-mass spectrometry (LC-MS) metabolomics analysis. Multivariate analysis including principal components analysis and partial least squares discriminant analysis, were applied to clearly separated the metabolites of infected and control rats. These metabolites included lipids, hormones, peptides, alkaloids and amino acids. Metabolic pathways, such as purine and pyrimidine metabolism, primary bile acid biosynthesis and glycerophospholipid metabolism were perturbed during the infection process.

Conclusions: These findings showed significant systemic metabolic perturbations of the liver in clonorchiasis. It also provided a new insight into the pathogenesis from the metabolic level, which might be helpful for making treatment strategies and prevention of clonorchiasis.

\section{Background}

Clonorchiasis, caused by Clonorchis sinensis (C. sinensis), is an important public health problem. It is estimated that about 35 million people are infected with $C$. sinensis worldwide [1]. Ingestion of freshwater fish contaminated with $C$. sinensis metacercariae is the scourer of infection. Chronic infection could cause several biological changes such as inflammation, epithelial hyperplasia, metaplasia and periductal fibrosis [2, 3]. Several hepatic abnormalities, including cholelithiases, cholecystitis, hepatic fibrosis, and even cholangiocarcinoma have been related to $C$. sinensis infection [4]. Our previous research had found that $C$. sinensis infection induced the dysregulation of host hepatic microRNA and hepatic apoptosis $[5,6]$. Recently, $C$. sinensis has been classified as a Group 1 carcinogen in humans by the International Agency for Research on Cancer [7]. However, the exact molecular pathogenic mechanism of clonorchiasis has not been fully illustrated.

Recently, omics techniques, including transcriptomics and proteomics, have been used to interrogate the interaction of host and parasite [8, 9]. Compared to the transcriptional or proteomic information, metabolomics could detect the simultaneous quantitation of many metabolites [10], reflect the physiological status and evaluate the disease pathogenesis [11]. Metabolomics had been used for the diagnosis, biomarker screening and studying the pathogenic mechanism $[12,13]$. The exploration of metabolites provide a global view of dynamic metabolic variations, which is helpful for underlying biochemical phenomena associated with the disease [14].

Metabolomics has been applied in parasitic diseases, such as Toxoplasma gondii, Trypanosoma brucei and onchocerciasis [15-17]. For example, Toxoplasma gondii infection induced significant metabolomic alterations and perturbed metabolic pathways in the spleen of infected mice [18]. The metabolomics and associated biochemical pathways could improve the understanding of the pathophysiological mechanism in parasitic diseases. Our previous studies also found $C$. sinensis infection caused hepatic iron metabolism abnormity [19]. However, there is no study investigating the global hepatic metabolic profiling or related metabolic pathways during $C$.sinensis infection. Especially, the function of some small-molecule metabolites in the host-parasite interactions has been poorly explained.

Therefore, this present study aimed to identify hepatic metabolic profiling and detect systemic metabolic pathways in $C$. sinensis infection animal models by a non-targeted liquid chromatography-mass spectrometry (LC-MS) metabolomics analysis (Figure 1). Our findings suggest that the metabolomics could provide a powerful approach for evaluating metabolic profiling and elucidating the pathophysiological mechanism in clonorchiasis.

\section{Results}

\section{Characteristics of $C$. sinensis infection in rats}

The $C$. sinensis eggs were detected in all infected groups by the Kato-Katz method. The egg detection rate could be detected at 4 weeks post infection $80 \%(16 / 20)$, and get to $100 \%(20 / 20)$ after 8 weeks post infection. The control group did not detected the eggs. Histopathological study of the livers from infected groups were characterized by epithelial hyperplasia, inflammatory infiltration of eosinophil and lymphocyte along the portal areas and hepatocytes hydropic degeneration (Fig. 2). The wall of bile ducts was thickened and the adult $C$. sinensis were found in some bile ducts. While, all control rats tissues did not present obvious pathological changes.

\section{Metabolite profiling of liver during $C$. sinensis infection}

The metabolite profiling in the rats liver during $C$. sinensis infection was present using by a non-targeted UHPLC-Q-TOF MS system. There were 11,922 and 11, 296 ions identified in each sample profile in $\mathrm{ESI}^{+}$and $\mathrm{ESI}^{-}$mode, respectively. After removing low-quality ions [relative standard deviation (RSD) > 30\%], 11902 and 11289 ions in each sample were recognized in $\mathrm{ESI}^{+}$and $\mathrm{ESI}^{-}$mode, respectively. To assess the reproducibility of our data sets, QC samples were measured, clustered closely to each other and separated from the tested samples. However, the PCA scores plots did not clearly differentiate infected groups from control group in Fig $3 \mathrm{~A}$ ( $\mathrm{ESI}^{+}$mode) and Fig $3 \mathrm{~B}$ (ESI ${ }^{-}$mode). The parameters R2 and Q2 confirmed the validity of the PLS-DA model as follows: $\mathrm{ESI}^{+}$mode, $\mathrm{R} 2 \mathrm{X}=0.577, \mathrm{Q} 2=0.0513$; $\mathrm{ESI}^{-}$mode, $\mathrm{R} 2 \mathrm{X}=0.405, \mathrm{Q} 2=0.0668$. Next, the PLS-DA analysis was performed and 
displayed in Fig $4 \mathrm{~A}$ ( $\mathrm{ESI}^{+}$mode) and Fig $4 \mathrm{~B}$ ( $\mathrm{ESI}^{-}$mode). The parameters R2 and Q2 confirmed the validity of the PLS-DA model as follows: ESI ${ }^{+}$mode, $\mathrm{R} 2 \mathrm{X}=0.567, \mathrm{R} 2 \mathrm{Y}=0.97, \mathrm{Q} 2=0.814 ; \mathrm{ESI}^{-}$mode, $\mathrm{R} 2 \mathrm{X}=0.608, \mathrm{R} 2 \mathrm{Y}=0.978, \mathrm{Q} 2=0.747$.

\section{Differential metabolites during different infection periods}

PCA was used to determine the global similarity/differences of samples in the study. As show in Figure 5 (ESI ${ }^{+}$mode) and Additional file 1: Figure S1 (ESI- mode), the control group was found distinctly to the left, whereas the infected group (8 weeks post infection) cluster slightly to the right without overlapping with the control group, respectively. The parameters R2 and Q2 confirmed the validity of the PCA model in the Additional file 2: Table S1. The clear separation was also constructed in the heatmap in Additional file 3: Figure S2 (ESI ${ }^{+}$mode) and Additional file 4: Figure S3 (ESI ${ }^{-}$mode). According to infection time, the metabolites form different infection groups were categorized clearly.

The significant differential metabolites involved in clonorchiasis were identified,

based on VIP > 1 and the $P$-value. An overview of identified altered metabolites detected both in negative and positive analysis mode with up- or downregulation had be presented in Additional file 5: Table S2. And then a Venn diagram was generated to visualize the common and unique metabolites between the infected groups (Figure 6). Especially, a total of 35 metabolites, included lipids, amino acids, peptides, antibiotics and alkaloids, were found common in the infected groups (Table 1). For example, choline and L-glutamine were significantly upregulated and cytosine, hydroxyproline were significantly decreased.

\section{Metabolic pathways affected by $C$.sinensis infection}

The biological pathways and roles involved in clonorchiasis were identified by MetaboAnalyst. As shown in Additional file 6: Table S3 and Additional file 7: Figure S4, Glycerophospholipid metabolism was found the most perturbed metabolic pathways among the infection groups, compared to the control group. While, other metabolic pathways included valine, leucine and isoleucine biosynthesis, Primary bile acid biosynthesis at 4 weeks post-infection group; linoleic acid metabolism and pyrimidine metabolism were identified at 8 weeks post-infection group. In addition, Arginine and proline metabolism, valine, leucine and isoleucine biosynthesis, purine and pyrimidine metabolism, alpha-Linolenic acid metabolism and glycerophospholipid metabolism involved in clonorchiasis were constructed a net-work in figure 7.

\section{Discussion}

Clonorchiasis is an important health problem worldwide [20]. However, the hepatic pathological mechanism of clonorchasis remained unclear. Metabolomics provide a powerful method to explore host-parasite interactions and discover the pathogenic mechanism at the biochemical level [21]. Recently, some studies had discovered endogenous metabolites and perturbed metabolic pathways in response to parasite infection [22]. However, it is still unclear whether $C$.sinensis infection cause metabolic change and dysregulation of biochemical pathways in the liver. In this study, a non-targeted UHPLC-Q-TOF MS method was applied to analysis the hepatic metabolite profiling and related metabolic pathways during $C$. sinensis infection. Theses results will be useful for exploring the pathophysiology mechanism, making the diagnosis and prevention therapies for clonorchiasis.

Firstly, this present study indicated that $C$.sinensis infection can induce systemic metabolic perturbations in the liver. Theses metabolites belonged to lipids, fatty acids, amino acids, products of amino acid metabolim, nucleotides. These metabolites might be a direct signal of $C$. sinensis activity or the consequence of the host response to the parasite. In addition, a total of 35 features/metabolites were shared in the infected groups. As shown in Table 1 , the levels of choline, L-glutamine, sn-glycerol-3-phosphoethanolamine increased, and cytosine, glycocholic acid, hydroxyproline were found decreased. These dynamic metabolites is not only the interplay between the host and $C$.sinensis, but also could perturb the biochemical profiles of both the parasite and host [23].

The identification and quantification of amino acids had provided significant insights into the pathogenesis of various diseases, such as cancer and arthritis [24]. In this study, we found significant changes of amino acids and eicosanoid metabolites during C.sinensis infection. These metabolites might been required by the parasites to support their growth [25, 26], and affect the host cell recruitment and proliferation during infection [27]. For example, leucine, as the branched-chain amino acids (BCAA), could provide energy when persistently energy consumption [28]. The up-regulate of leucine indicated C.sinensis need more energy to grow up. As an essential precursor for gluconeogenesis in the liver [29], the elevated level of alanine might indicate the impaired hepatic gluconeogenesis in clonorchiasis. While, S-Methyl-5'-thioadenosine participate the cysteine and methionine metabolism, as important participator in the tricarboxylic acid (TCA) cycle [30]. The decreased of S-Methyl-5'-thioadenosine indicated decreasing metabolic TCA cycle activity by the parasite. Glutamine is catabolized via TCA cycle and generatesy-aminobutyric acid, which are essential for the growth of C.sinensis [31].

Additionally, our results also revealed that purine and pyrimidine metabolism were involved in clonorchiasis. Hydroxyproline origin from the posttranslational hydroxylation of proline in proteins (primarily collagen) [31]. Both proline and hydroxylation have important metabolic, physiological and structural functions in collagens [32]. The decreased levels of hydroxyproline suggested that $C$.sinensis might facilitate its release from the liver cell of host.

The other identified significantly differential metabolites were lipids, including PC(6:0/6:0), alpha-linolenic acid (ALA), linolenic acid and glycerophosphocholine. Except the primary components of biological membranes, lipids also participate in signal recognition and transmission [33]. $C$. 
sinensis could utilize lipids for energy or physical requirements to survive longer, by up-regulating expression and enzymatic activity of CsACAT (an enzyme involved in fatty acid metabolism). The higher survival rate of adult worms was also reported in an environment with a higher level of lipids [34]. Therefore, the dysregulation of lipid metabolism suggested that the parasite modulated host cell environment to survival and used lipids to complete membrane biogenesis [35].

Because $C$.sinensis lack the oxygen-dependent pathways to synthesize fatty acids, it has to depend on its host for complex lipids [36]. For example, alpha-linolenic acid (ALA), an essential omega-3 fatty acid, is commonly found in seeds, nuts, and vegetable oils [37]. However, because ALA is an essential fatty acid (i.e., cannot be synthesized by the rat), its increased level must been derived from the rat diet. As to arachidonic acid (the main polyunsaturated fatty acid), it is released and metabolized to a series of eicosanoids, including the inflammatory leukotrienes and prostaglandins [38]. The up-regulation of arachidonic acid and its inflammatory metabolites mediated the regulation of cell survival, chemotaxis, migration, apoptosis and mitogenesis [39].

With regard to the metabolic pathway, linoleic acid metabolism and glycerophospholipid metabolism were found involved in clonorchiasis. Especially in glycerophospholipid metabolism, glycerophospholipids compose the main lipid category of mammalian cell membranes [40], which play a vital role in regulating transport processes, protein function and signal transduction [41]. Additionally, glycerophospholipids are also essential components of lipoproteins and influence their function and metabolism [33]. The perturbed in these metabolites pathway play biological roles relevant to host-parasite interaction.

This study applies highly sophisticated technology to understand the impact of a neglected parasite on metabolism of the host. However this study has several limitations. Firstly, the main limitation was that samples were not collected from the control group over time. Thus, a larger samples with timematched control groups should be assessed in future studies. Secondly, we only investigated metabolic changes in the liver. The serum or other tissue (spleen) might also be useful to address the pathogenesis of clonochiasis. Therefore, we will investigate the other tissue using metabolomics method, in order to explore the parasite-host interaction. Finally, the identified differential metabolites may likely participate in the pathogenesis of clonorchiasis, but the underlying molecular mechanisms still needs further investigations to reveal them.

\section{Conclusion}

Based on a non-targeted LC-MS/MS metabolomics method, we provide a new insight into the pathogenesis of clonorchiasis from the metabolic level. These differential metabolites mainly involved lipids (glycerophosphocholine) and amino acid (leucin and L-glutamine). Several critical metabolic pathways might play a great biological roles on the host-parasite interaction, including glycerophospholipid metabolism, purine and pyrimidine metabolism and primary bile acid biosynthesis. Theses results will be helpful for understanding the pathogenesis of clonorchiasis. In future, supplemental assays should be done to further investigate the metabolic liver alterations.

\section{Methods}

\section{Parasites, animals and infection}

C. sinensis metacercariae were collected from Pseudorasbora parva captured in the Songhuajiang River of Heilongjiang Province. The process of collection and preparation is performed as described previously [42]. In brief, the fish were placed in an ice box at $0^{\circ} \mathrm{C}$ and transported to the laboratory. Next, the fish were washed with tap water, disrupted in a Waring Blender and digested with a pepsin- $\mathrm{HCl}(0.6 \%)$ artificial gastric juice, at $37^{\circ} \mathrm{C}$ for $12 \mathrm{~h}$. Then, the digested mixture was passed through three sieves with the mesh size of $1000 \mu \mathrm{m}, 300 \mu \mathrm{m}$ and $106 \mu \mathrm{m}$. After centrifugation, mass pure metacercariae were collected and kept in $0.1 \mathrm{M}$ phosphate-buffered saline (PBS, pH 7.4) at $4^{\circ} \mathrm{C}$ until used.

Wistar rats (150g, 5-6 weeks old, male) were purchased from the Harbin Medical University Laboratory Animal Center. There was a rat in a single animal cage. The rats were housed with an inverse 12 hours day-night cycle with lights on at $8: 30 \mathrm{pm}$ in a temperature $\left(20 \pm 1^{\circ} \mathrm{C}\right)$ and humidity $(55 \pm 5 \%)$ controlled room, and fed on standard laboratory food (CS-101; Liaoning Changsheng biotechnology co., Ltd) and water.

All the rats were randomly divided into 3 groups, 4 weeks post infection (wpi), 8 wpi and control groups. Animal model experiment were carried out in the afternoon. In the infection groups, the rats were randomly selected and individually infected orally with 50 metacercariae ( $\mathrm{n}=10 \mathrm{per}$ group). The KatoKatz method was used to detect $C$. sinensis eggs from the rat feces [43]. Briefly, KK smears were prepared using $41.7 \mathrm{mg}$ of feces, and the EPG (eggs per gram of feces) was determined by multiplying the number of eggs per smear by 24 . Control group rats $(n=10)$ were fed with $50 \mu l$ of sterile normal solutio.

At 4 wpi and 8 wpi, the rats were sacrificed under ether anesthesia by cervical dislocation and livers were quickly removed and rinsed with saline solution $(0.9 \% \mathrm{NaCl} \mathrm{w} / \mathrm{v})$, and stored at $-80^{\circ} \mathrm{C}$ for analysis. And then livers from control rats were collected at 8 wpi. Inhalation anesthesia (ether anesthesia) reduced animal suffering and increased the success rate of anesthesia. Briefly, every rat was put into a glass anesthesia box containing several ethersoaked cotton balls $(2-4 \mathrm{ml})$. After slow breathing, decreased limb tensioning and dull corneal response, the rats were sacrificed by cervical dislocation.

For histology, liver samples were fixed by $4 \%$ paraformaldehyde, then dehydrated in a graded series of ethanol and embedded in paraffin wax. Sections were made $(5 \mu \mathrm{m})$ using a microtome, then stained with hematoxylin-eosin (H\&E) and examined under an optical microscope (Olympus, Tokyo, Japan).

\section{Chemicals}


LC-MS-grade water ( $\mathrm{H} 20)$, acetonitrile (ACN), methanol (MeOH), $0.1 \%$ formic acid (FA) in water and $0.1 \%$ FA in ACN were purchased from Honeywell (Muskegon, MI, USA). Ammonium acetate (NH4AC), ammonium hydroxide ( $\mathrm{NH} 4 \mathrm{OH}$ ), ammonium fluoride (NH4F) were purchased from Sigma-Aldrich (St. Louis, MO, USA) and dissolved in LC-MS-grade water prior to use.

\section{Sample collection and preparation}

A total of $100 \mathrm{mg}$ liver sample was add $1 \mathrm{~mL}$ of cold methanol/acetonitrile/H2O (2:2:1,v/v/v). The samples were vortexed for 30 seconds and repeated 5 times. After using MP FastPrep 24 machine (MP Biomedicals, USA) to homogenize the lysate $(24 \times 2,6.0 \mathrm{M} / \mathrm{S}, 60 \mathrm{~s}$, twice), the mixture was sonicated for 30 minutes two times at low temperature, and centrifuged by Eppendorf $5430 \mathrm{R}$ for $15 \mathrm{~min}\left(15,000 \mathrm{~g}, 4^{\circ} \mathrm{C}\right)$, and then dried in a vacuum centrifuge at $1500 \mathrm{~g}$ overnight. The samples were redissolved in $100 \mu \mathrm{L}$ acetonitrile/water $(1: 1, \mathrm{v} / \mathrm{v})$ solvent.

\section{UHPLC-Q-TOF MS analysis}

Analyses were performed using an UHPLC (1290 Infinity LC, Agilent Technologies) with a quadrupole time-of-flight mass spectroscopy (AB Sciex Triple TOF 5600) in Shanghai Applied Protein Technology Co., Ltd.

In the HILIC separation process, we utilized a $2.1 \mathrm{~mm} \times 100 \mathrm{~mm}$ ACQUIY UPLC BEH $1.7 \mu \mathrm{m}$ column (waters, Ireland) to analysis the samples. The mobile phase contained $A=25 \mathrm{mM}$ ammonium acetate and $25 \mathrm{mM}$ ammonium hydroxide in water and $\mathrm{B}=$ acetonitrile. The gradient was set at $85 \% \mathrm{~B}$ ( 1 min), reduced to $65 \%$ in $11 \mathrm{~min}$, reduced to $40 \%$ in $0.1 \mathrm{~min}$ (hold on $4 \mathrm{~min}$ ), increased to $85 \%$ in $0.1 \mathrm{~min}$, and then re-balanced in $5 \mathrm{~min}$.

The ESI source situation were used as below: Curtain gas (CUR) as 30, source temperature: $600^{\circ} \mathrm{C}$, lon Source Gas 1 (Gas1) as 60 , lon Source Gas2 (Gas2) as 60 , lonSpray Voltage Floating (ISVF) $\pm 5500 \mathrm{~V}$. The instrument was set over the $\mathrm{m} / \mathrm{z}$ range $60-1000 \mathrm{Da}$, with the accumulation time of TOF MS scan (0.20 s/spectra) in MS analysis. Over the m/z range $25-1000 \mathrm{Da}$, product ion scan was set at $0.05 \mathrm{~s} / \mathrm{spectra}$ in auto MS/MS acquisition. With the advantages of high sensitivity mode, information dependent acquisition (IDA) was applied to acquire the product ion scan. The condition was set as below: the collision energy (CE) was set at $35 \pm 15 \mathrm{eV}$; Declustering Potential (DP), $60 \mathrm{~V}(+)$ and $60 \mathrm{~V}(-)$; exclude isotopes within $4 \mathrm{Da}$, candidate ions to monitor per cycle: 10

During mass spectra collection, samples were placed in automatic sampler at $4^{\circ} \mathrm{C}$. To monitor the stability and repeatability of analytical system, quality control (QC) samples were prepared by pooling $10 \mu \mathrm{L}$ of each sample and injected prior to analysis. And then blank and QC samples were injected every 5 samples injections throughout the analytical run.

\section{Data processing}

The raw MS data (Wiff format files) were converted to mzXML format using ProteoWizard (http://proteowizard.sourceforge.net/) and processed using $\mathrm{R}$ package XCMS (version 3.2). For peak picking, the parameters were set: centWave $\mathrm{m} / \mathrm{z}=25 \mathrm{ppm}$, peak width $=\mathrm{c}(10,60)$, prefilter $=\mathrm{c}(10,100)$. For peak grouping, $\mathrm{bw}=5$, mzwid $=0.025$, minfrac $=0.5$ were used. $\mathrm{R}$ i836 3.1.1 was used to run XCMS and Collection of Algorithms of

Metabolite Profile Annotation (CAMERA, 1.42.0). The code was used to perform data processing showed in supporting information. The parameters was loaded on supporting information. The variables with more than $50 \%$ of the nonzero measurement values in at least one group were identified.

Compound identification of metabolites was performed by comparing the accuracy of $\mathrm{m} / \mathrm{z}$ values ( $<25 \mathrm{ppm})$, and MS/MS spectra were interpreted with an in house database (Shanghai Applied Protein Technology Co., Ltd.) established with authentic standards.

\section{Statistical analysis}

After normalized to total peak intensity, the processed data were uploaded into SIMCA-P (version 14.1, Umetrics, Umea, Sweden). The data were meancentered and Pareto-scaled before multivariate statistical analysis. PCA, an unsupervised multivariate statistical method, could provide an overview of all observations in data tables, such as groupings, trends, and outliers of the different groups of samples. Then, the supervised partial least-squares discriminant analysis (PLS-DA) were applied to discriminate the different variables between groups. A default seven-fold cross-validation and testing with 200 random permutations were used to avoid the over-fitting of supervised PLS-DA models. The goodness-of-fit parameters for the PLS model, R2 and Q2, were calculated which varied from 0 to 1. The values of R2 and Q2 parameters were used to verify the fitness and predictive ability of the model. The variable importance in the projection (VIP) value was applied to clarify its contribution to the classification in the PLS-DA model. Fold change (FC) was set to be $\geq 1.2$ or $\leq 0.8333$. Log2 FC based on metabolite abundance was used to demonstrate how the differential abundance of liver metabolites varied at different stages of infection groups.

Heatmaps were employed to describe the unbalanced metabolic profiling among C.sinensis infected and control rats. Euclidean distance algorithm for similarity measure and average linkage clustering algorithm (clustering uses the centroids of the observations) for clustering were selected when performing hierarchical clustering. Based on the deferentially expressed metabolite data (log2-scaled), heatmaps were structured by the MultiExperiment Viewer (MeV) v. 4.9 software (http:// mev.tm4.org/).

The Pathway analysis of the differentially expressed metabolites was depended on KEGG database (http://www.genome.jp/kegg/) and MetaboAnalyst 3.0 (http://www.MetaboAnalyst.ca/). According to our research background and the pathway enrichment results, the corresponding KEGG pathways were extracted. The analyses were applied based on the fisher' exact test, considering the metabolites as background data sets. And only pathways with FDR-values under a threshold of 0.05 were considered as significant. The identified pathways influenced by $C$.sinensis infection compared to controls 
are presented according to $\mathrm{p}$-values from the pathway enrichment analysis (y-axis) and pathway impact values from pathway topology analysis ( $\mathrm{x}$-axis), with the most impacted pathways colored in red color.

\section{Abbreviations}

LC-MS: liquid chromatography-mass spectrometry; UHPLC-QTOF MS: ultra high performance liquid tandem chromatography quadrupole time of flight mass spectrometry; PCA: principal component analysis; PLS-DA: partial least-squares discriminant analysis; VIP: variable importance in the projection; FC: Fold change; RSD: relative standard deviation; ESI: positive ion mode; ALA: alpha-linolenic acid

\section{Declarations}

\section{Ethics approval and consent to participate}

The animal research protocol has been reviewed and assigned by the Medical Ethics Review Committee of Harbin Medical University that approved our animal experiments. All animal experiments were carried out on the basis of the Guide for the Care and Use of Laboratory Animals published by the Ministry of Science and Technology of the People's Republic of China. In the study, we made great efforts to reduce animal suffering and the number of animals.

\section{Consent for publication}

All authors have given their permission to publish data presented in this manuscript.

\section{Availability of data and materials}

All the data supporting our findings is contained within the manuscript.

\section{Competing interests}

The authors declare that they have no competing interests.

\section{Funding}

This work was supported by National Natural Science Foundation of China (81971958, 81601785 and 81401684), Natural Science of Heilongjiang province (H2017024), Post-doctoral Science Foundation (LBH-Q174). Heilongjiang province College students innovative entrepreneurial training program (201710226044). Harbin medical university College students innovative entrepreneurial training program $(201810226003,201810226027)$. The funders had no role in study design, data collection and analysis, decision to publish, or preparation of the manuscript.

\section{Authors' contributions}

Conceptualization: Xiaoli Zhang, Ruifeng Wang, Su Han. Data curation: Xiaoli Zhang, Beibei Sun, Rui Chen. Formal analysis: Beibei Sun, Rui Chen, Xinyi Hu. Investigation: Beibei Sun, Xinyi Hu, Yannan Gao, Shanshan Duan, Jing Fang, Ping Wang, Xinshu Zhao. Resources: Xiaoli Zhang, Ruifeng Wang, Su Han. Methodology: Ruifeng Wang, Beibei Sun, Rui Chen, Ping Wang, Xinyi Hu, Yannan Gao, Shanshan Duan, Xinshu Zhao. Writing-original draft: Xiaoli Zhang, Ruifeng Wang, Su Han. Writing-review \& editing: Xiaoli Zhang, Su Han.

\section{Acknowledgements}

We thank the staff of laboratory animal research center of Harbin Medical University for their cooperation and support.

\section{References}

1. Lun ZR, Gasser RB, Lai DH, Li AX, Zhu XQ, Yu XB, Fang YY. Clonorchiasis: a key foodborne zoonosis in China. Lancet Infect Dis. 2005;5(1):31-41.

2. Hong ST, Fang Y. Clonorchis sinensis and clonorchiasis, an update. Parasitol Int. 2012;61(1):17-24.

3. Nam JH, Moon JH, Kim IK, Lee MR, Hong SJ, Ahn JH, Chung JW, Pak JH. Free radicals enzymatically triggered by Clonorchis sinensis excretorysecretory products cause NF-KB-mediated inflammation in human cholangiocarcinoma cells. Int J Parasitol. 2012;42(1):103-113.

4. Tang ZL, Huang Y, Yu XB. Current status and perspectives of Clonorchis sinensis and clonorchiasis: epidemiology, pathogenesis, omics, prevention and control. Infect Dis Poverty. 2016;5(1):016-0166.

5. Zhang X, Jin Z, Da R, Dong Y, Song W, Chen X, Huang Q, Ling H, Che Y, Li Y et al. Fas/FasL-dependent apoptosis of hepatocytes induced in rat and patients with Clonorchis sinensis infection. Parasitol Res. 2008;103(2):393-399.

6. Han S, Tang Q, Lu X, Chen R, Li Y, Shu J, Zhang X, Cao J. Dysregulation of hepatic microRNA expression profiles with Clonorchis sinensis infection. BMC Infect Dis. 2016;16(1):016-2058.

7. Han S, Zhang X, Chen R, Wen J, Li Y, Shu J, Ling H, Zhang F. Trends in prevalence of clonorchiasis among patients in Heilongjiang province, Northeast China (2009-2012): implications for monitoring and control. PLoS One. 2013;8(11). 
8. Pak JH, Moon JH, Hwang SJ, Cho SH, Seo SB, Kim TS. Proteomic analysis of differentially expressed proteins in human cholangiocarcinoma cells treated with Clonorchis sinensis excretory-secretory products. J Cell Biochem. 2009;108(6):1376-1388.

9. Young ND, Jex AR, Cantacessi C, Campbell BE, Laha T, Sohn WM, Sripa B, Loukas A, Brindley PJ, Gasser RB. Progress on the transcriptomics of carcinogenic liver flukes of humans-unique biological and biotechnological prospects. Biotechnol Adv. 2010;28(6):859-870.

10. Olszewski KL, Morrisey JM, Wilinski D, Burns JM, Vaidya AB, Rabinowitz JD, Llinás M. Host-parasite interactions revealed by Plasmodium falciparum metabolomics. Cell Host Microbe. 2009;5(2):191-199.

11. Wang X, Zhao X, Chou J, Yu J, Yang T, Liu L, Zhang F. Taurine, glutamic acid and ethylmalonic acid as important metabolites for detecting human breast cancer based on the targeted metabolomics. Cancer Biomark. 2018;23(2):255-268.

12. Liu L, Wang M, Yang X, Bi M, Na L, Niu Y, Li Y, Sun C. Fasting serum lipid and dehydroepiandrosterone sulfate as important metabolites for detecting isolated postchallenge diabetes: serum metabolomics via ultra-high-performance LC-MS. Clin Chem. 2013;59(9):1338-1348.

13. Liu L, Li Y, Guan C, Li K, Wang C, Feng R, Sun C. Free fatty acid metabolic profile and biomarkers of isolated post-challenge diabetes and type 2 diabetes mellitus based on GC-MS and multivariate statistical analysis. J Chromatogr B Analyt Technol Biomed Life Sci. 2010;878(28):2817-2825

14. Chen R, Han S, Liu X, Wang K, Zhou Y, Yang C, Zhang X. Perturbations in amino acids and metabolic pathways in osteoarthritis patients determined by targeted metabolomics analysis. J Chromatogr B Analyt Technol Biomed Life Sci. 2018;15:54-62.

15. Zhou CX, Zhou DH, Elsheikha HM, Liu GX, Suo X, Zhu XQ. Global Metabolomic Profiling of Mice Brains following Experimental Infection with the Cyst-Forming Toxoplasma gondii. PLoS One. 2015;10(10).

16. Lamour SD, Alibu VP, Holmes E, Sternberg JM. Metabolic Profiling of Central Nervous System Disease in Trypanosoma brucei rhodesiense Infection. $\mathrm{J}$ Infect Dis. 2017;216(10):1273-1280.

17. Bennuru S, Lustigman S, Abraham D, Nutman TB. Metabolite profiling of infection-associated metabolic markers of onchocerciasis. Mol Biochem Parasitol. 2017;215:58-69.

18. Chen XQ, Zhou CX, Elsheikha HM, He S, Hu GX, Zhu XQ. Profiling of the perturbed metabolomic state of mouse spleen during acute and chronic toxoplasmosis. Parasit Vectors. 2017;10(1):017-2282.

19. Han S, Tang Q, Chen R, Li Y, Shu J, Zhang X. Hepatic iron overload is associated with hepatocyte apoptosis during Clonorchis sinensis infection. BMC Infect Dis. 2017;17(1):017-2630.

20. Qian MB, Utzinger J, Keiser J, Zhou XN. Clonorchiasis. Lancet. 2016;387(10020):800-810.

21. Ghosh S, Sengupta A, Sharma S, Sonawat HM. Metabolic fingerprints of serum, brain, and liver are distinct for mice with cerebral and noncerebral malaria: a ${ }^{1}$ H NMR spectroscopy-based metabonomic study. J Proteome Res. 2012;11(10):4992-5004.

22. Zhou CX, Cong W, Chen XQ, He SY, Elsheikha HM, Zhu XQ. Serum Metabolic Profiling of Oocyst-Induced Toxoplasma gondii Acute and Chronic Infections in Mice Using Mass-Spectrometry. Front Microbiol. 2018;8(2612).

23. Abdelrazig S, Ortori CA, Davey G, Deressa W, Mulleta D, Barrett DA, Amberbir A, Fogarty AW. A metabolomic analytical approach permits identification of urinary biomarkers for Plasmodium falciparum infection: a case-control study. Malar J. 2017;16(1):017-1875

24. Zhou Y, Zhang X, Chen R, Han S, Liu Y, Liu X, Gao M, Yang C, Lu D, Sun B et al. Serum amino acid metabolic profiles of ankylosing spondylitis by targeted metabolomics analysis. Clin Rheumatol. 2020;4(10):020-04974.

25. Marino ND, Boothroyd JC. Toxoplasma growth in vitro is dependent on exogenous tyrosine and is independent of AAH2 even in tyrosine-limiting conditions. Exp Parasitol. 2017;176:52-58.

26. Silva NM, Rodrigues CV, Santoro MM, Reis LF, Alvarez-Leite JI, Gazzinelli RT. Expression of indoleamine 2,3-dioxygenase, tryptophan degradation, and kynurenine formation during in vivo infection with Toxoplasma gondii: induction by endogenous gamma interferon and requirement of interferon regulatory factor 1 . Infect Immun. 2002;70(2):859-868.

27. Li Y, Xiao W, Luo W, Zeng C, Deng Z, Ren W, Wu G, Lei G. Alterations of amino acid metabolism in osteoarthritis: its implications for nutrition and health. Amino Acids. 2016;48(4):907-914

28. Holecek M. The BCAA-BCKA cycle: its relation to alanine and glutamine synthesis and protein balance. Nutrition. 2001;17(1):00483-00484.

29. Snell K. Muscle alanine synthesis and hepatic gluconeogenesis. Biochem Soc Trans. 1980;8(2):205-213.

30. Xu Y, Quan CS, Jin X, Jin X, Zhao J, Jin L, Kim JS, Guo J, Fan S, Ha NC. Crystallization and preliminary X-ray diffraction analysis of the interaction of Aeromonas hydrophila MtaN-1 with S-adenosylhomocysteine. Acta Crystallogr F Struct Biol Commun. 2015;71(Pt 4):393-396.

31. Le A, Lane AN, Hamaker M, Bose S, Gouw A, Barbi J, Tsukamoto T, Rojas CJ, Slusher BS, Zhang H et al. Glucose-independent glutamine metabolism via TCA cycling for proliferation and survival in B cells. Cell Metab. 2012;15(1):110-121.

32. Li P, Wu G. Roles of dietary glycine, proline, and hydroxyproline in collagen synthesis and animal growth. Amino Acids. 2018;50(1):29-38.

33. Ecker J, Liebisch G. Application of stable isotopes to investigate the metabolism of fatty acids, glycerophospholipid and sphingolipid species. Prog Lipid Res. 2014;54:14-31.

34. Lin J, Qu H, Chen G, He L, Xu Y, Xie Z, Ren M, Sun J, Li S, Chen W et al. Clonorchis sinensis acetoacetyl-CoA thiolase: identification and characterization of its potential role in surviving in the bile duct. Parasit Vectors. 2015;8(125):015-0728.

35. Gupta N, Zahn MM, Coppens I, Joiner KA, Voelker DR. Selective disruption of phosphatidylcholine metabolism of the intracellular parasite Toxoplasma gondii arrests its growth. J Biol Chem. 2005;280(16):16345-16353.

Page $7 / 14$ 
36. Huang L, Hu Y, Huang Y, Fang H, Li R, Hu D, Li W, Li X, Liang C, Yu X. Gene/protein expression level, immunolocalization and binding characteristics of fatty acid binding protein from Clonorchis sinensis (CsFABP). Mol Cell Biochem. 2012;363(1-2):367-376.

37. Jovanovski E, Li D, Thanh Ho HV, Djedovic V, Ruiz Marques AC, Shishtar E, Mejia SB, Sievenpiper JL, de Souza RJ, Duvnjak L et al. The effect of alpha-linolenic acid on glycemic control in individuals with type 2 diabetes: A systematic review and meta-analysis of randomized controlled clinical trials. Medicine. 2017;96(21):0000000000006531.

38. Greene ER, Huang S, Serhan CN, Panigrahy D. Regulation of inflammation in cancer by eicosanoids. Prostaglandins Other Lipid Mediat. 2011;96(14):27-36.

39. Levick SP, Loch DC, Taylor SM, Janicki JS. Arachidonic acid metabolism as a potential mediator of cardiac fibrosis associated with inflammation. J Immunol. 2007;178(2):641-646.

40. van Meer G, de Kroon Al. Lipid map of the mammalian cell. J Cell Sci. 2011;124(Pt 1):5-8.

41. Wymann MP, Schneiter R. Lipid signalling in disease. Nat Rev Mol Cell Biol. 2008;9(2):162-176.

42. Fan PC. Viability of metacercariae of Clonorchis sinensis in frozen or salted freshwater fish. Int J Parasitol. 1998;28(4):603-605.

43. Hong ST, Choi MH, Kim CH, Chung BS, Ji Z. The Kato-Katz method is reliable for diagnosis of Clonorchis sinensis infection. Diagn Microbiol Infect Dis. 2003;47(1):345-347.

\section{Tables}

Table 1. list of the significant differential metabolites in the liver during Clonorchis sinensis infection. 


\begin{tabular}{|c|c|c|c|c|c|c|c|c|c|}
\hline \multirow[t]{2}{*}{ Metabolites } & \multirow{2}{*}{$\begin{array}{l}\text { lon } \\
\text { mode }\end{array}$} & \multirow[t]{2}{*}{$\mathrm{m} / \mathrm{z}$} & \multirow[t]{2}{*}{$\mathrm{RT}(\mathrm{s})$} & \multicolumn{3}{|l|}{$4 w$} & \multicolumn{3}{|l|}{$8 w$} \\
\hline & & & & VIP & $\mathrm{FC}$ & $P$-value & VIP & $\mathrm{FC}$ & $P$-value \\
\hline $\begin{array}{l}\text { all cis-(6,9,12)- } \\
\text { Linolenic acid }\end{array}$ & $\begin{array}{l}\text { ESI } \\
(+)\end{array}$ & 279.2317 & 75.672 & 2.73225 & 0.758612561 & 0.009448743 & 2.10974 & 0.773842006 & 0.009711669 \\
\hline $\begin{array}{l}\text { All-trans-retinoic acid } \\
\text { (Vitamin A acid) }\end{array}$ & $\begin{array}{l}\text { ESI } \\
(+)\end{array}$ & 301.21597 & 74.888 & 2.91149 & 0.591838765 & 0.000170085 & 1.66843 & 0.731540885 & 0.016019382 \\
\hline $\begin{array}{l}\text { Anthranilic acid } \\
\text { (Vitamin L1) }\end{array}$ & $\begin{array}{l}\text { ESI } \\
(+)\end{array}$ & 138.05511 & 495.334 & 4.95363 & 0.79631447 & 0.00130446 & 3.12278 & 0.858957558 & 0.012929772 \\
\hline Choline & $\begin{array}{l}\text { ESI } \\
(+)\end{array}$ & 104.1074 & 474.993 & 9.16767 & 1.450231162 & 0.002673271 & 8.87087 & 1.540289174 & 0.000358098 \\
\hline Cytosine & $\begin{array}{l}\text { ESI } \\
(+)\end{array}$ & 112.05077 & 411.379 & 1.95201 & 0.878008016 & 0.028896604 & 2.00943 & 0.822130981 & 0.000733933 \\
\hline Deoxyadenosine & $\begin{array}{l}\text { ESI } \\
(+)\end{array}$ & 252.10861 & 241.029 & 1.12288 & 0.743678903 & 0.001498489 & 1.27625 & 0.589838845 & 6.77E-07 \\
\hline Glycocholic acid & $\begin{array}{l}\mathrm{ESI} \\
(+)\end{array}$ & 430.2952 & 402.612 & 1.93696 & 0.475427121 & 0.010617156 & 1.36668 & 0.514790641 & 0.024010933 \\
\hline Hydroxyproline & $\begin{array}{l}\text { ESI } \\
(+)\end{array}$ & 114.05506 & 453.669 & 2.27718 & 0.406058576 & 0.000962204 & 2.20149 & 0.293504365 & 0.000142058 \\
\hline $\begin{array}{l}\text { Indole-2-carboxylic } \\
\text { acid }\end{array}$ & $\begin{array}{l}\mathrm{ESI} \\
(+)\end{array}$ & 162.05468 & 43.995 & 1.28821 & 2.361583789 & $9.58 \mathrm{E}-05$ & 1.0913 & 2.221242734 & $1.64 \mathrm{E}-06$ \\
\hline Isopentenyladenosine & $\begin{array}{l}\text { ESI } \\
(+)\end{array}$ & 336.16645 & 107.672 & 1.88373 & 0.487507057 & 0.001864155 & 1.36514 & 0.547586635 & 0.005167746 \\
\hline L-Glutamine & $\begin{array}{l}\text { ESI } \\
(+)\end{array}$ & 147.0762 & 710.1265 & 1.68907 & 1.432330976 & 0.016268191 & 1.63725 & 1.583657578 & 0.004001807 \\
\hline $\begin{array}{l}\text { Linoleoyl } \\
\text { ethanolamide }\end{array}$ & $\begin{array}{l}\text { ESI } \\
(+)\end{array}$ & 324.28965 & 58.517 & 1.06276 & 0.778919895 & 0.036937224 & 1.82477 & 0.528336458 & 0.000294341 \\
\hline L-Leucine & $\begin{array}{l}\text { ESI } \\
(+)\end{array}$ & 132.10188 & 450.5 & 5.00514 & 0.807497317 & 0.038356154 & 4.10908 & 0.807964789 & 0.025599824 \\
\hline $\begin{array}{l}\text { Palmitoyl } \\
\text { ethanolamide }\end{array}$ & $\begin{array}{l}\text { ESI } \\
(+)\end{array}$ & 300.28937 & 59.177 & 1.07834 & 0.785640935 & 0.001940064 & 1.39096 & 0.657167861 & 1.91E-05 \\
\hline $\begin{array}{l}\text { S-Methyl-5'- } \\
\text { thioadenosine }\end{array}$ & $\begin{array}{l}\mathrm{ESI} \\
(+)\end{array}$ & 298.09698 & 169.444 & 13.8546 & 0.629787484 & 0.004838671 & 13.869 & 0.560765306 & 0.000818364 \\
\hline $\begin{array}{l}\text { sn-Glycerol 3- } \\
\text { phosphoethanolamine }\end{array}$ & $\begin{array}{l}\mathrm{ESI} \\
(+)\end{array}$ & 216.06299 & 707.431 & 3.23237 & 1.254981162 & 0.048671204 & 3.52469 & 1.388254393 & 0.008682199 \\
\hline Taurocholate & $\begin{array}{l}\mathrm{ESI} \\
(+)\end{array}$ & 498.28859 & 337.003 & 3.00319 & 1.457215847 & 0.049301323 & 2.45452 & 1.391940649 & 0.024234046 \\
\hline Val-Ile & $\begin{array}{l}\text { ESI } \\
(+)\end{array}$ & 231.1699 & 335.424 & 1.15501 & 1.6083819 & 0.00156135 & 1.46846 & 2.234693919 & 0.02144037 \\
\hline Val-Ala & $\begin{array}{l}\mathrm{ESI} \\
(+)\end{array}$ & 189.1228 & 77.112 & 1.539 & 1.418591561 & 0.044672185 & 2.34642 & 2.115245511 & 0.045291101 \\
\hline Uracil & $\begin{array}{l}\text { ESI } \\
(+)\end{array}$ & 113.0346 & 145.483 & 1.45277 & 0.694213442 & 6.72577E-05 & 1.10812 & 0.705856865 & 7.77206E-05 \\
\hline L-Pipecolic acid & $\begin{array}{l}\mathrm{ESI} \\
(+)\end{array}$ & 130.08613 & 537.962 & 1.49732 & 1.829935947 & 0.00607913 & 1.29932 & 1.6822452 & 0.0003519 \\
\hline Ile-Ala & $\begin{array}{l}\text { ESI } \\
(+)\end{array}$ & 203.13847 & 74.379 & 2.03177 & 1.411574411 & 0.037034449 & 2.73358 & 1.835771628 & 0.000695882 \\
\hline His-lle & $\begin{array}{l}\text { ESI } \\
(+)\end{array}$ & 269.16075 & 514.977 & 1.03143 & 1.396591727 & 0.026368031 & 1.37835 & 1.934063788 & 0.04513821 \\
\hline Argininosuccinic acid & $\begin{array}{l}\text { ESI } \\
(+)\end{array}$ & 291.12951 & 850.626 & 1.43032 & 1.290801415 & 0.029349786 & 1.21154 & 1.279507993 & 0.022250118 \\
\hline 7-Oxocholesterol & $\begin{array}{l}\mathrm{ESI} \\
(+)\end{array}$ & 401.34155 & 55.114 & 1.67515 & 0.690414822 & 0.0023886 & 1.66313 & 0.62581625 & 0.001368047 \\
\hline Alpha-D-Glucose & $\begin{array}{l}\text { ESI } \\
(-)\end{array}$ & 179.05654 & 493.179 & 2.46119 & 0.710599294 & 0.003034608 & 2.61295 & 0.628709688 & 0.002339324 \\
\hline Eicosapentaenoic acid & $\begin{array}{l}\text { ESI } \\
(-)\end{array}$ & 301.21503 & 69.3715 & 1.99045 & 0.895705351 & 0.3889767 & 5.22078 & 0.783592053 & 0.000915527 \\
\hline
\end{tabular}




\begin{tabular}{|c|c|c|c|c|c|c|c|c|c|}
\hline Myristic acid & $\begin{array}{l}\text { ESI } \\
(-)\end{array}$ & 227.20097 & 73.894 & 5.35593 & 1.144974188 & 0.048246327 & 5.47587 & 1.156270582 & 0.043728265 \\
\hline pregnenolone sulfate & $\begin{array}{l}\text { ESI } \\
(-)\end{array}$ & 395.18541 & 40.112 & 4.64259 & 0.435465253 & 0.000321747 & 4.91205 & 0.368418482 & 4.90E-06 \\
\hline Xanthosine & $\begin{array}{l}\text { ESI } \\
(-)\end{array}$ & 283.06671 & 373.7045 & 2.58849 & 0.768426403 & 0.000116929 & 1.84599 & 0.843383926 & 0.00837643 \\
\hline Pantetheine & $\begin{array}{l}\text { ESI } \\
(-)\end{array}$ & 277.12081 & 67.913 & 1.18415 & 0.750849187 & 0.01094792 & 1.49072 & 0.646251192 & 0.004002172 \\
\hline Linoleic acid & $\begin{array}{l}\text { ESI } \\
(-)\end{array}$ & 279.23162 & 70.669 & 10.611 & 0.935948241 & 0.033358382 & 14.4381 & 0.842850431 & 0.000385108 \\
\hline D-Ribose & $\begin{array}{l}\text { ESI } \\
(-)\end{array}$ & 209.06646 & 501.277 & 1.67129 & 0.480478507 & 0.003504703 & 1.46061 & 0.516987967 & 0.004657167 \\
\hline D-gluconate & $\begin{array}{l}\text { ESI } \\
(-)\end{array}$ & 195.05121 & 635.8795 & 2.84971 & 0.739800344 & 0.001387005 & 2.58154 & 0.757147079 & 0.000513459 \\
\hline Capric acid & $\begin{array}{l}\text { ESI } \\
(-)\end{array}$ & 171.13938 & 77.042 & 2.23532 & 1.526730891 & 0.010192217 & 1.799 & 1.473658412 & 0.043211676 \\
\hline
\end{tabular}

RT, Retention time; VIP, variable importance for projection; FC, Fold change;

\section{Figures}

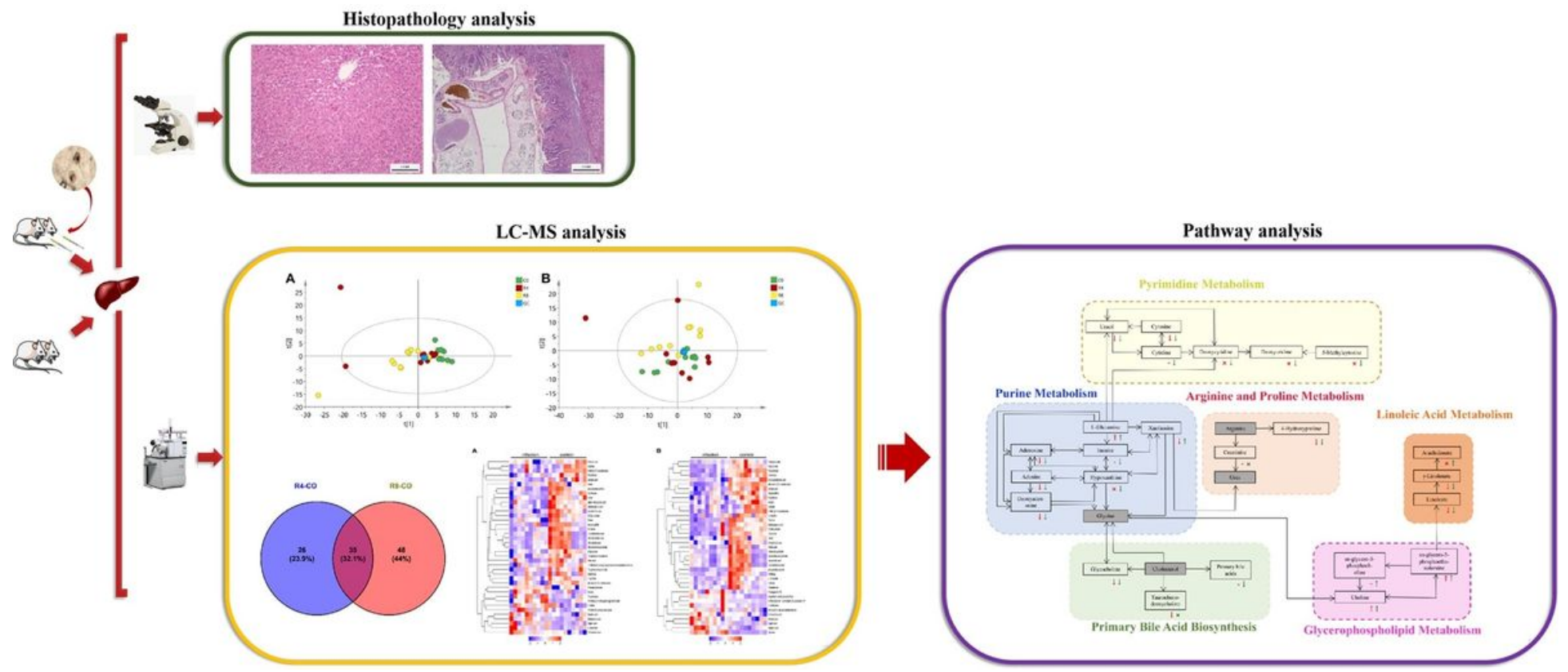

Figure 1

Experimental flow chart. 

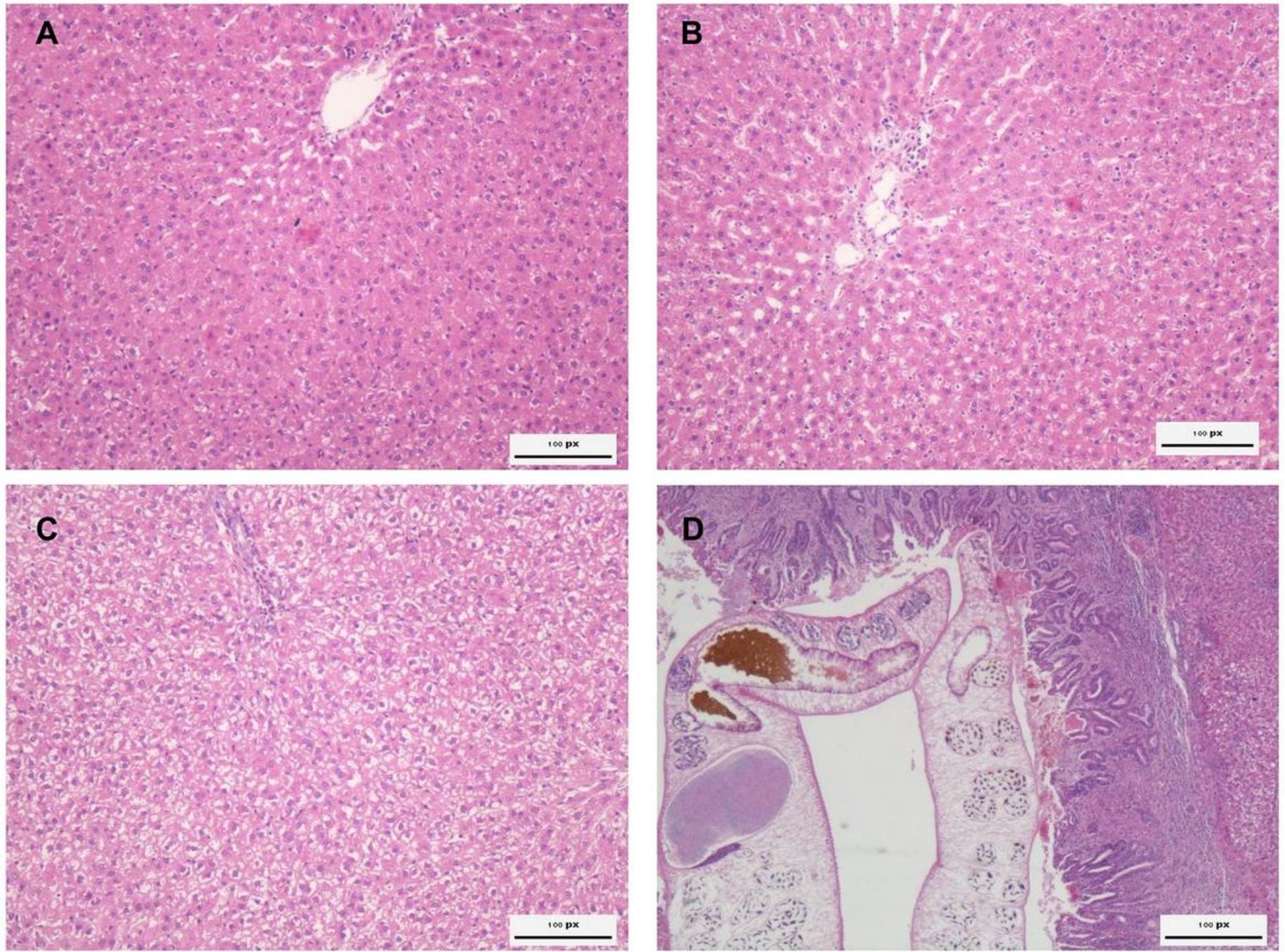

\section{Figure 2}

Histopathological changes in the liver tissues from C.sinensis-infected rats. (A) Hepatic tissue from control, control rats shows a normal histological structure; (B) At 4 weeks-post infection, liver showed multifocal mononuclear cell aggregations (mainly lymphocytes) with some vacuolated hepatocyte; (C) At 8 weeks-post infection, liver showed mild mononuclear cellular infiltration and the enlarged and swollen hepatocytes; (D) The adults of C.sinensis in the liver. Histopathological analysis was conducted on livers from control and C.sinensis -infected rats at 4 and 8 weeks post infection.

Photomicrographs of liver tissue sections stained with hematoxylin and eosin (original magnification, $\times 100$ ).
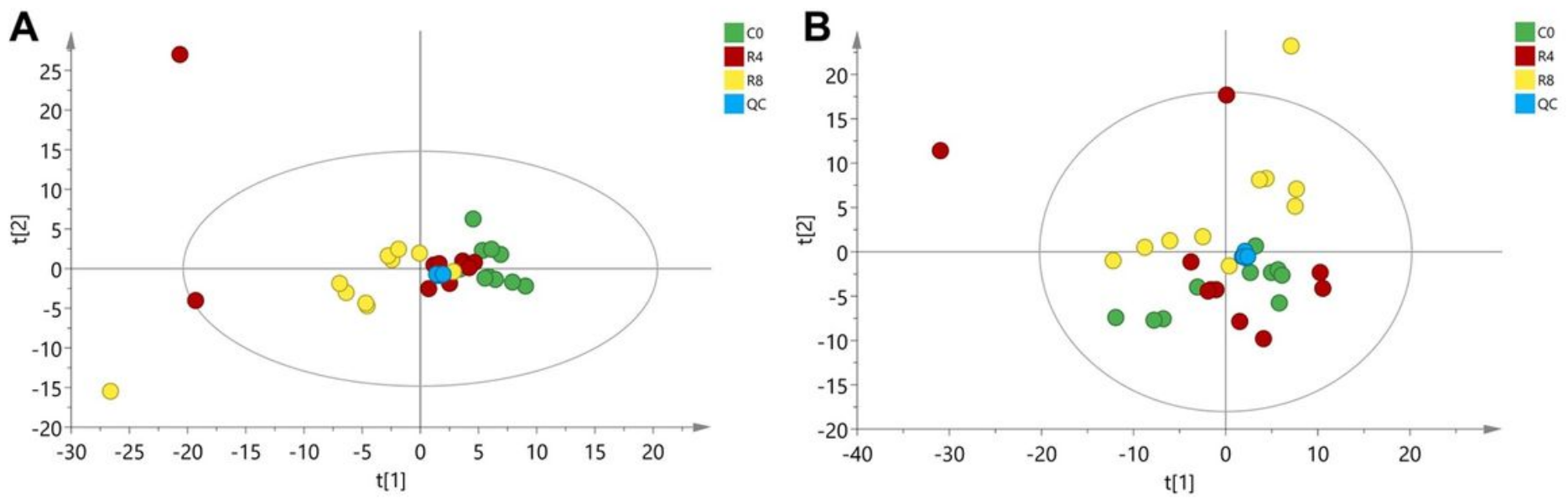

Figure 3 
PCA score plots fingerprints of hepatic metabolites during C. sinensis infection. (A) PCA scores plot of hepatic metabolites in the positive ion mode (ESI+); (B) PCA scores plot of hepatic metabolites in the negative ion mode (ESI-); R4, 4 weeks-post infection; R8, 8 weeks-post infection; C0, healthy control; QC, quality control.
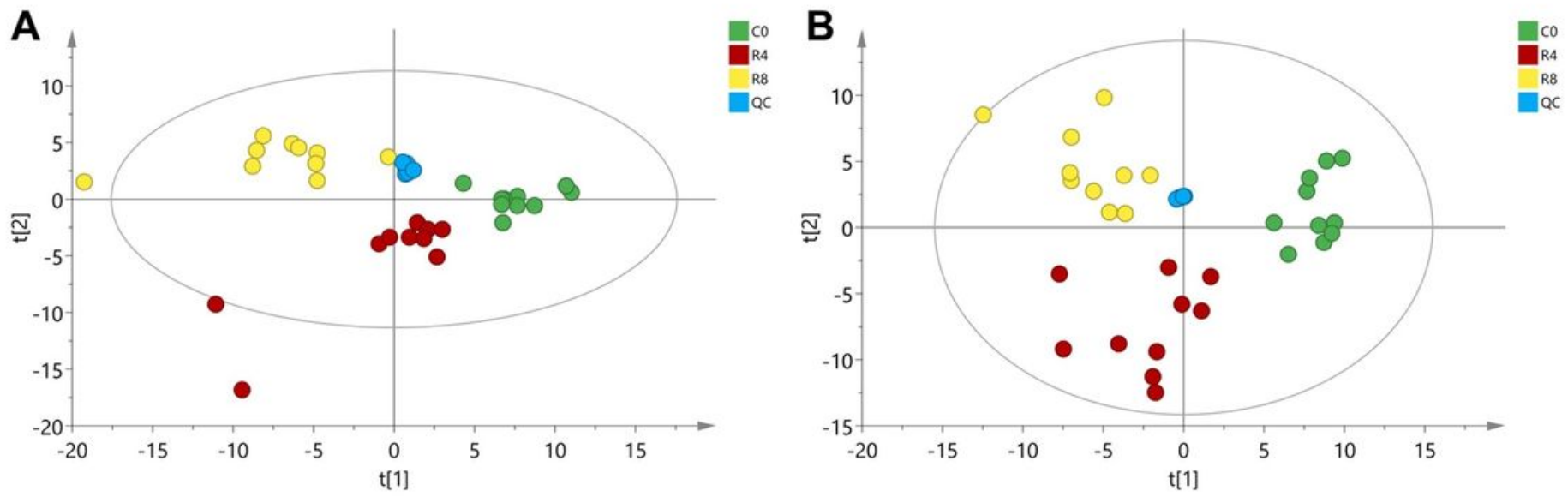

\section{Figure 4}

PLS-DA score plots of hepatic metabolites of the four groups. (A) PLS-DA scores plot of hepatic metabolites in the positive ion mode (ESI+); (B) PLS-DA scores plot of hepatic metabolites in the negative ion mode (ESI-); R4, 4 weeks-post infection, R8, 8 weeks-post infection, C0, healthy control; QC, quality control.
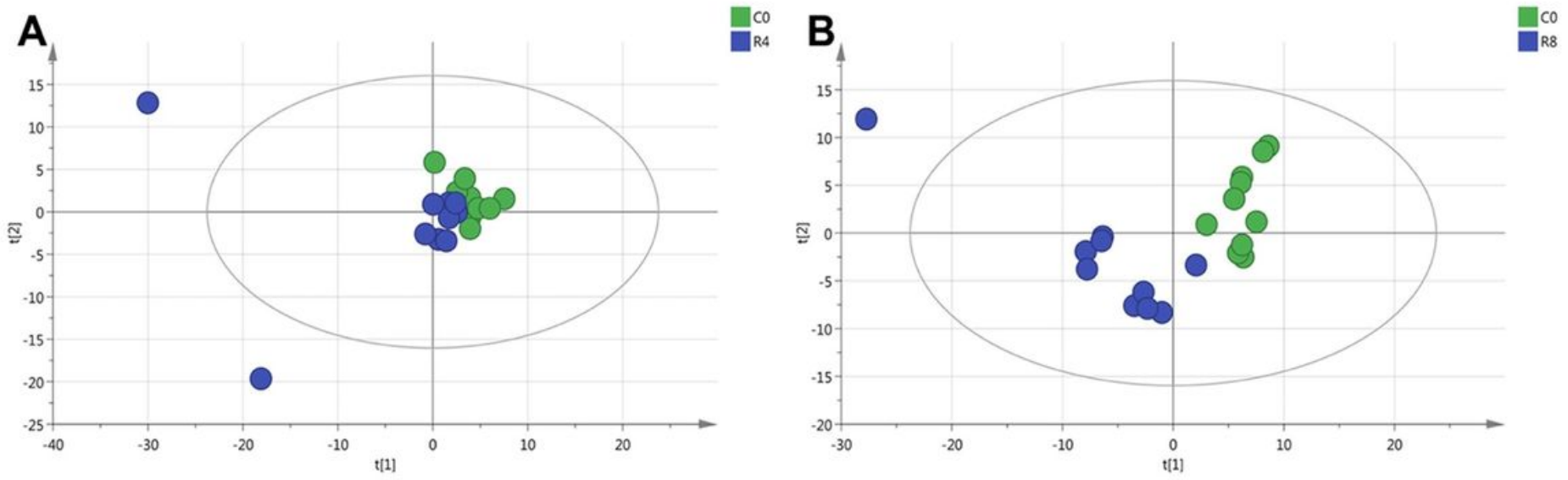

Figure 5

PCA separating differential metabolites of infected any time point vs. control groups in ESI+ mode. (A) R4 vs C0, (B) R8 vs C0; R4, 4 weeks-post infection, R8, 8 weeks-post infection, C0, healthy control. 


\section{R4-CO}

\section{R8-CO}

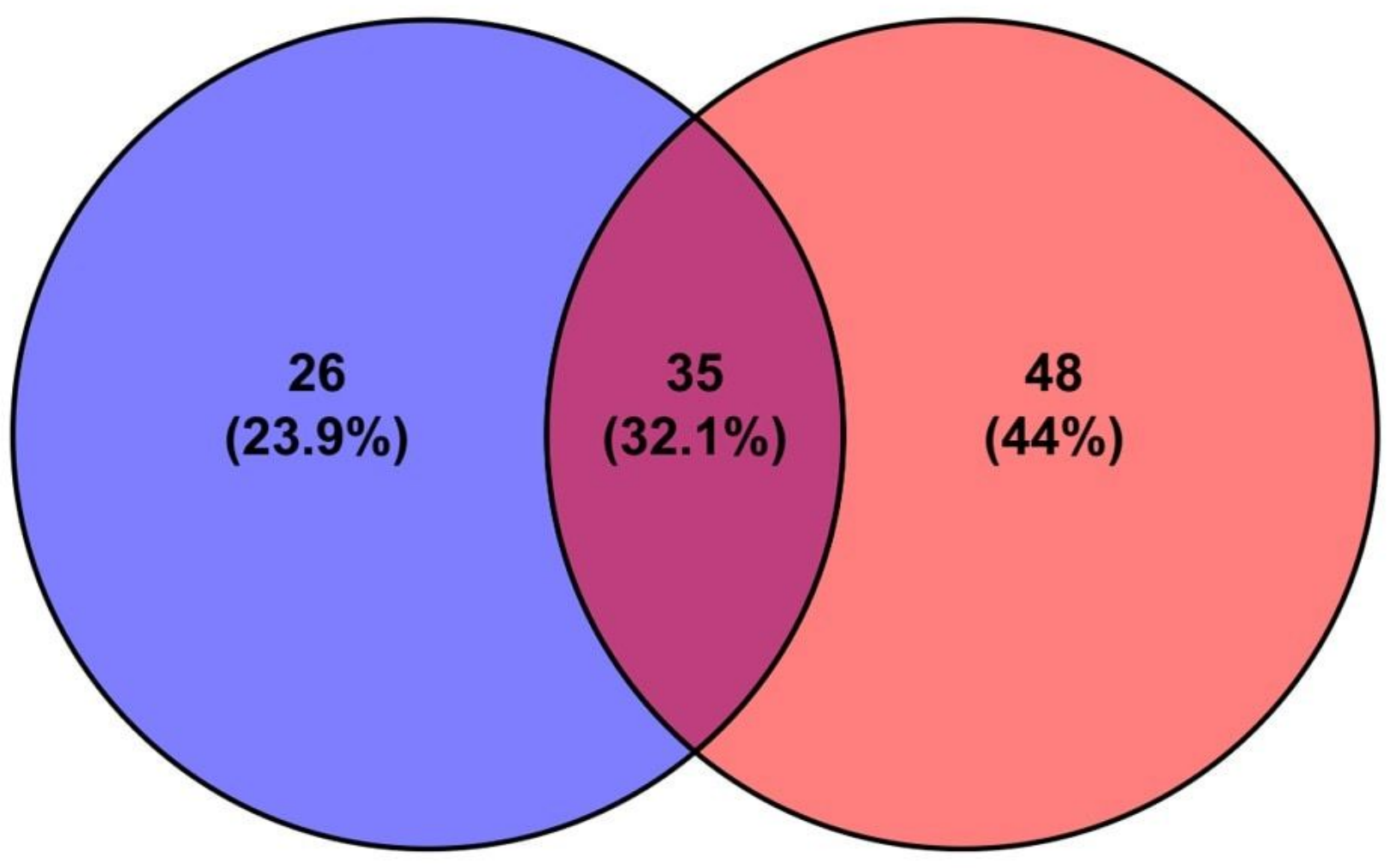

\section{Figure 6}

Venn diagram displaying the common and unique metabolites between two group. There were 35 significantly differential metabolites were shared in infected groups. R4, 4 weeks-post infection, R8, 8 weeks-post infection, C0, healthy control. 


\section{Pyrimidine Metabolism}

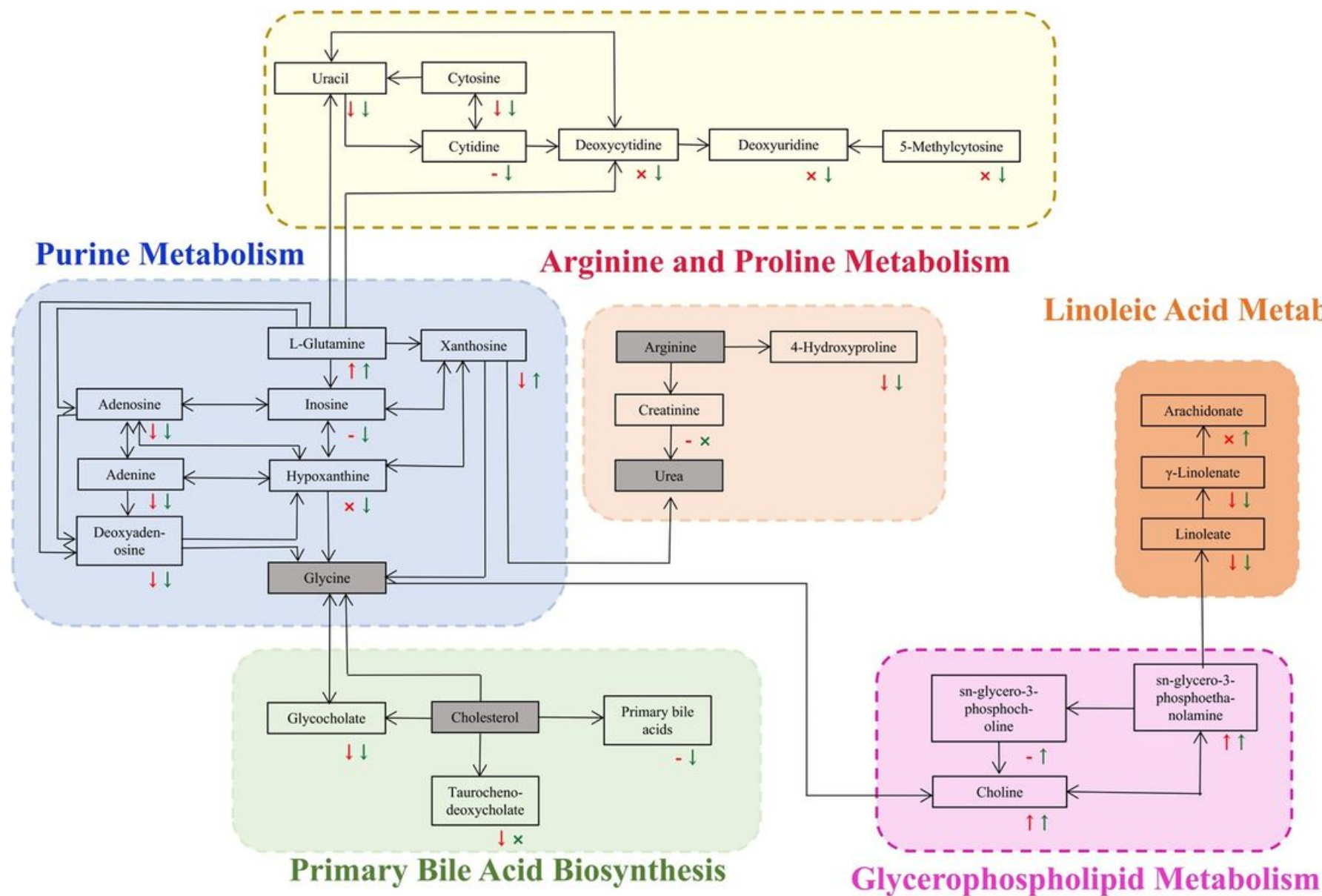

Figure 7

Summary the perturbed metabolic pathways during the two infection groups. Up and down arrow means metabolites change trend. The horizontal line indicates no change. The cross sign indicates no detection. 4 weeks-post infection (red), 8 weeks-post infection (green).

\section{Supplementary Files}

This is a list of supplementary files associated with this preprint. Click to download.

- Additionalfile7FIGS42020.6.7.tif

- Additionalfiles256TableS1S2S3.xls

- Additionalfile4FIGS3heatmapNEG2020.6.7.tif

- Additionalfile3FIGS2heatmapPOS2020.6.7.tif

- Additionalfile1FIGS1PCAXtwogoupsNEG2020.6.7.tif 